\title{
Evaluation of enamel surface roughness using different types of polishing system after orthodontic
}

\author{
bracket debonding
}

\begin{abstract}
Maryam Fouad Bilal ${ }^{*}$, Lanja Abubakir Ali ${ }^{*}$, Deman Hasan Hamid ${ }^{*}$, Rebin Ali Mohammed Amin ${ }^{*}$
Background: returning surface of the enamel to its pre-orthodontic state after debonding of brackets without any damage to the texture of enamel is a clinical contest. removal of Residual adhesive using correct and suitable tools and methods ensures a smooth surface and healthy plaque-free environment. Therefore; the study aims to determine the safest method to finish enamel surface after bracket debonding using three different methods.

Method: Thirty premolars extracted for orthodontic purposes were selected for this study. The samples were coded 1-30 randomly and surface roughness was measured before bracket placement using profilometer. Then bracket bonded in the middle third of the buccal surface of the premolars and then debonded using debonding plier. The sample was divided into three groups, 10 for each group (group 1: 18-flute tungsten carbide bur, group 2: 12- flute tungsten carbide bur, group 3: adhesive removing plier). Then the second roughness measurement was recorded.

Result: It is found that debonding with adhesive removing plier was the least efficient method followed by 12 -fluted tungsten carbide bur, so the best clean-up method in this study achieved is by using 18-fluted tungsten carbide bur.

Conclusion: The 18-fluted flame-shaped tungsten carbide bur at high speed for orthodontic adhesive removal demonstrated more favorable results in our hands, as it resulted in the smoothest enamel surface and could reasonably be used as a standard by which future other burs or other procedures are compared.
\end{abstract}

Keywords: 12-fluted tungsten carbide bur, 18-fluted tungsten carbide bur, adhesive removing plier, profilometer, enamel surface roughness.

* College of Dentistry, Hawler Medical University, Erbil, Iraq. Correspondence: mariam.bilal@hmu.edu.krd. 07504495649.

\section{Introduction}

After finishing fixed orthodontic treatment, one of the most important points is to keep enamel surface untouched during adhesive removal and polishing. The ideal would be minimal enamel loss at each stage of bonding, debonding, and enamel cleanup process and the production of an enamel surface with the same degree of roughness or smoothness as original, untreated teeth. ${ }^{1}$ Etching enamel surface enhances adhesion between the bonding agent and enamel, but removing brackets and improper remnant adhesive removal at the end of orthodontic treatment is a reason to cause iatrogenic enamel injury, excessive accumulation of plaque, irritation of gingival tissue and staining of teeth. ${ }^{2}$ The investigation of an effective and harmless method of removing adhesive resin after the debonding of brackets and tubes is subject of interest of many orthodontists and researchers, followed by the production of a different type of instruments and innovation of new procedures. Remaining adhesive on the enamel surface following bracket debonding can be removed in different ways, including manual instruments (pliers and scalers), various shape and design of tungsten carbide burs of 8 to 30 -fluted configuration with low 
or high-speed handpieces, soflex discs, ultrasonic devices, air abrasion units, and lasers, however, some studies have shown that some of the recommended methods hurt enamel surface. ${ }^{3}$. So many orthodontists use their own methods of removing resin according to trial and error without knowledge of real damage. They may be hurting to the enamel surface. ${ }^{4}$

Therefore, the purpose of this study was to assess enamel surface roughness following different resin removal after bracket debonding. The assessment was carried out by means of the profilometer. Moreover, the present study also aimed to compare enamel surface roughness before and after bracket debonding.

\section{Materials and methods}

Thirty premolars extracted for orthodontic purpose were selected for this study with no history of previous fixed orthodontic therapy, enamel cracks, caries, restorations, surface irregularities or, roughness. The teeth were cleaned and stored in normal saline at room temperature. ${ }^{5}$ One investigator conducted all experiments to remove variability between operator techniques. ${ }^{6}$

The tooth was embedded in a rectangular mold (about $25 \mathrm{~mm}$ width and $35 \mathrm{~mm}$ length) longitudinally which, is filled with stone only the buccal surface of the crown was seen (Figure 1).

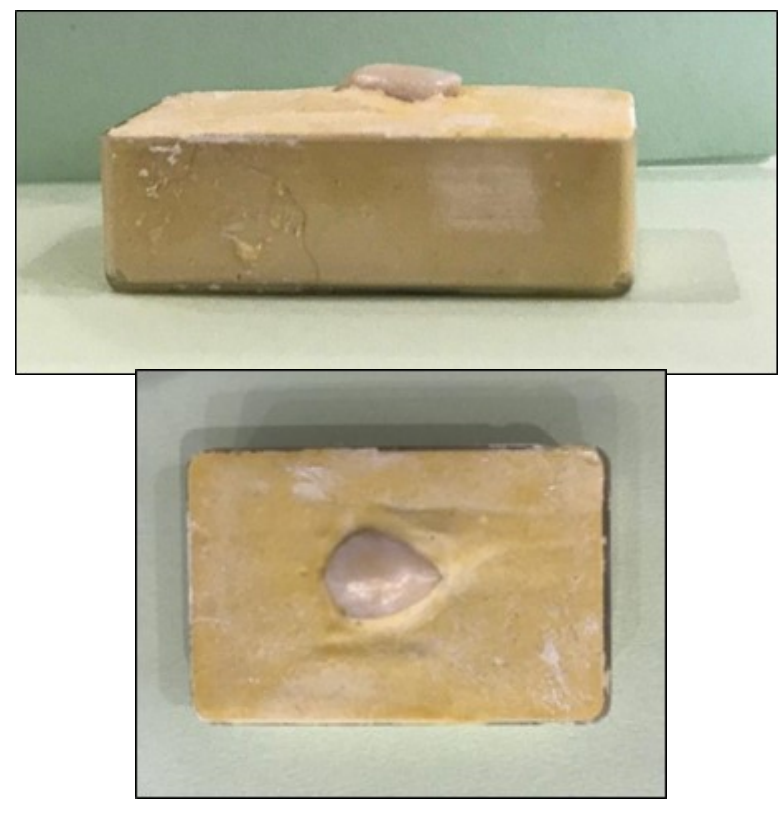

Figure 1: Teeth embedded in the stone.
Thirty-five teeth were examined using a profilometer device to assess whether the teeth have already roughness or not, five teeth were excluded and, 30 teeth were involved in the study according to the inclusion criteria of the study.

The buccal surface of each tooth was polished using non-fluoridated pumice with a rubber cup attached to a low-speed handpiece for 10 seconds, then each tooth was washed with water spray for 10 seconds, and dried with air spray for 10 seconds. The middle third of the enamel on the buccal surfaces of the teeth was etched with $37 \%$ phosphoric acid gel (3M Unitek) for 30 seconds, rinsed for 30 seconds, and dried with air spray for 10 seconds. The bonding agent (3M Unitek) was applied on the buccal

The samples were coded from 1 to 30 for identification purposes and, the middle of the buccal surface was subjected to profilometric analysis to obtaining enamel surface roughness parameters before any treatment $\left(1^{\text {st }} \text { roughness recording }\right)^{7}$ (Figure 2).

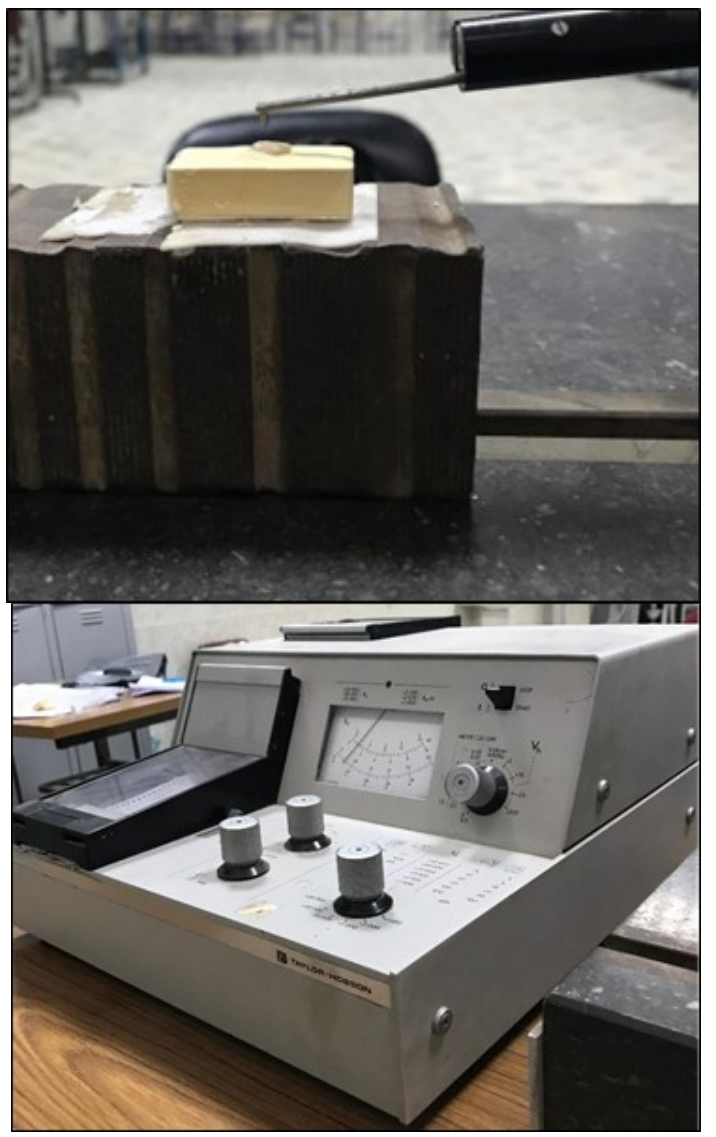

Figure 2: Profilometer device. 
surface by brush, then tooth subjected to air spray to remove the excess of bonding then light-cured. ${ }^{5}$ The adhesive (3M Unitek) placed on the base of the brackets $(022$ Roth $\backslash 3 \mathrm{M}$ Unitek) and, the brackets were placed in the middle third of the teeth in occlusal-gingival and mesial-distal directions, and seated with firm pressure. ${ }^{1}$ The excess adhesive resin was carefully removed with an explorer. The curing time was 20 seconds, 10 seconds mesially and, 10 seconds distally as manufactures instruction. ${ }^{1}$ The samples were immersed in normal saline at room temperature for two days (Figure 3).

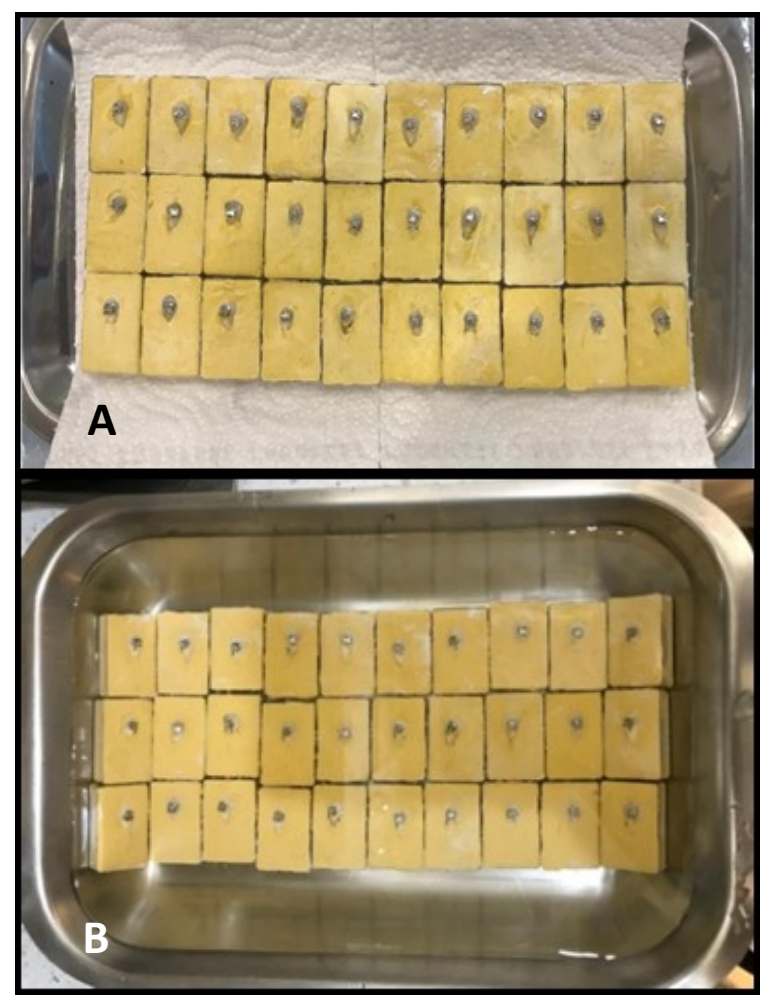

Figure 3: a) Samples with brackets bonded in place. b) Samples were immersed in normal saline

After two days the blocks were removed from normal saline and, the debonding of the brackets was carried out by debonding plier (MEDESY3000/82) by applying a gentle squeezing force on the outer wings of the bracket by the same operator ${ }^{1}$ in mesiodistal direction. ${ }^{8}$ After debonding of brackets samples were divided into 3 groups, each group ten based on the type of clean-up method applied for the removal of remaining adhesive resin left after deboning of the bracket which is as follow: Group 1: 18 fluted carbide adhesive removal bur by reliance orthodontic products (\#118S/Ref. RSB) \USA, on the high-speed turbine.

Group 2: 12 fluted carbide adhesive removal bur by prima classic (FG H22016ALGK) $\backslash \mathrm{UK}$, on the high-speed turbine. Group 3: adhesive removal plier by AO (001-344RTE) \USA 10B256021 002.

The remaining adhesive resins were removed in group $1 \&$ group 2 by using high-speed turbines with water coolant and, a new bur was used for each tooth and, the teeth were finished until the adhesive was cleaned. The conventional adhesive removing plier was used to clean up the enamel surface in group 3 (Figure 4,5).

After remnant adhesive removal, a new assessment of enamel surface roughness was obtained by using profilometer analysis.

Statistical Analysis. Data were entered and analyzed by the Statistical Package for Social Sciences (SPSS, version 22). The paired t-test was used to compare the means of enamel roughness before and after the bonding of the brackets. One-way Analysis of Variance (ANOVA) was used to compare the means of the differences between the reading (after - before) among the three study groups. A post hoc test (LSD) was used to compare every two means (after doing the ANOVA test). A 'P value' of less than 0.05 was considered as statistically significant.

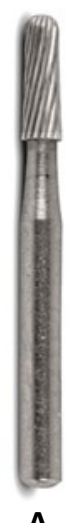

A

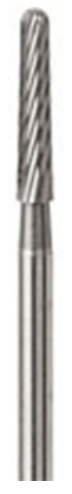

B

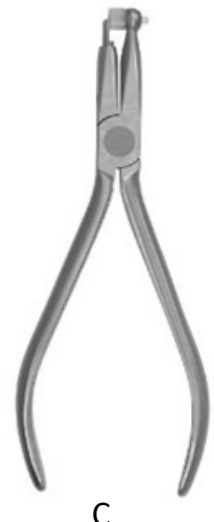

C
Figure 4: a) 18-flude tungusten carbide bur

b) 12-flute tungusten carbide bur c) adhesive removing plier 


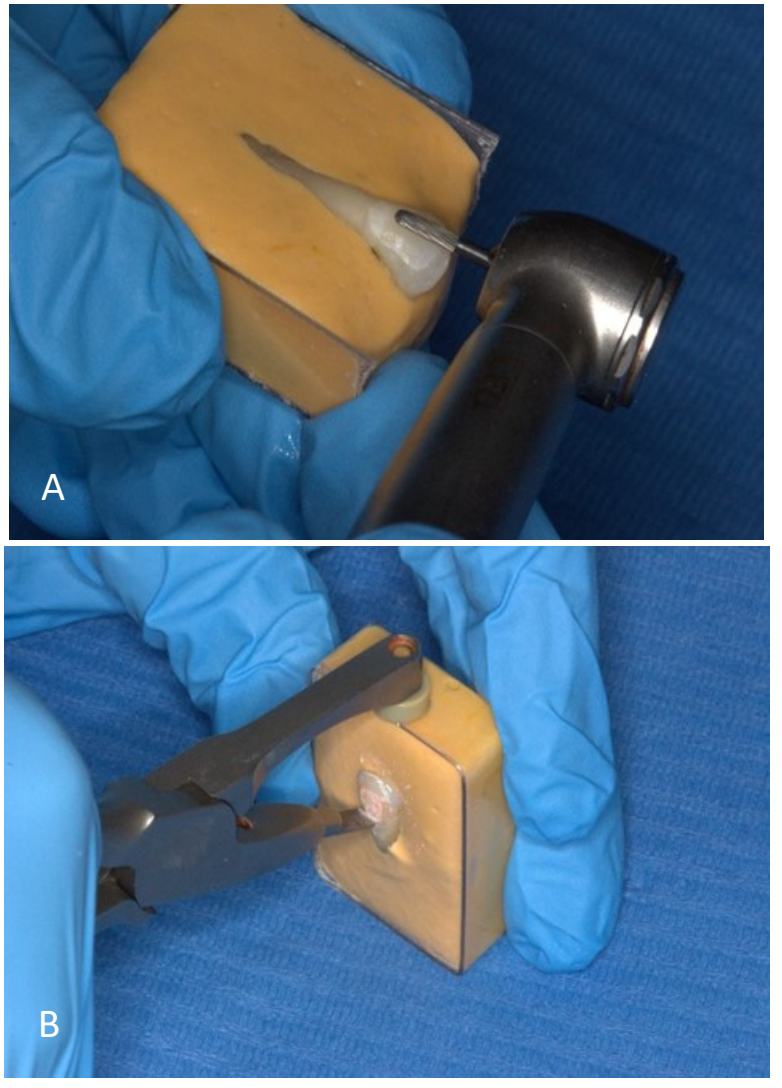

Figure 5: Removing adhesive by a) tungsten carbide bur. b) adhesive removing plier

\section{Results}

A total of 30 teeth (premolar) were collected and randomly distributed into three groups (ten samples for each group). Each group subjected to profilometric measurements to measure the enamel roughness before bracket bonding. Then second roughness recording was carried out after bracket debonding and resin removal by three different methods. Finishing of the enamel

surface was done in the first group by using 18 flute tungsten carbide burs, in the second group by using 12 flute tungsten carbide bur $\&$ third group by using adhesive removing plier.

There was a highly statistically significant difference between the means of enamel roughness measured before and after bracket placement in all the three study groups $(\mathrm{P}<0.001)$, as showed in table 1 .

The enamel surface roughness was significantly increased after bracket debonding and finishing of the enamel surface by three different methods.

ANOVA test showed no statistically significant difference $(\mathrm{P}=0.107)$ in the means of difference between the three groups but the LSD (Least Significant Difference) test showed significant dif

ference between group one (18 fluted tungsten carbide bur) and group three (adhesive removing plier $)(\mathrm{P}=0.043)$ were it's evident in table 2 that the mean difference of group one was $0.0382 \mu \mathrm{m}$ which was significantly less than mean difference of group three which was $0.511 \mu \mathrm{m}$.

Table 1. Means of enamel roughness $(\mu \mathrm{m})$ before and after the bonding of orthodontic brackets

\begin{tabular}{|c|c|c|c|c|c|}
\hline \multirow[b]{3}{*}{ Groups } & \multicolumn{4}{|c|}{ Enamel roughness $(\mu \mathrm{m})$} & \multirow[b]{3}{*}{$\mathbf{P}$} \\
\hline & \multicolumn{2}{|c|}{ Before bracket placement } & \multicolumn{2}{|c|}{ After bracket placement } & \\
\hline & Mean & $( \pm S D)$ & Mean & $( \pm S D)$ & \\
\hline Group 1 & 0.029 & $( \pm 0.003)$ & 0.410 & $( \pm 0.111)$ & $<0.001$ \\
\hline Group 2 & 0.030 & $( \pm 0.004)$ & 0.445 & $( \pm 0.135)$ & $<0.001$ \\
\hline Group 3 & 0.029 & $( \pm 0.006)$ & 0.540 & $( \pm 0.156)$ & $<0.001$ \\
\hline
\end{tabular}


Table 2. Means of the differences (after - before) in the enamel roughness $(\mu \mathrm{m})$ among the three study groups.

\begin{tabular}{|c|c|c|c|c|c|c|}
\hline Groups & $\mathbf{N}$ & $\begin{array}{c}\text { Mean difference } \\
\text { (after - before) }\end{array}$ & $\mathbf{( \pm S D )}$ & P (ANOVA) & LSD groups & P (LSD) \\
\hline Group 1 & 10 & 0.382 & $( \pm 0.111)$ & 0.107 & $\mathrm{G} 1 \times \mathrm{G} 2$ & 0.584 \\
\hline Group 2 & 10 & 0.415 & $( \pm 0.137)$ & & $\mathrm{G} 1 \times \mathrm{G3}$ & 0.043 \\
\hline Group 3 & 10 & 0.511 & $( \pm 0.158)$ & & $\mathrm{G} 2 \times \mathrm{G3}$ & 0.128 \\
\hline Total & 30 & 0.436 & $( \pm 0.143)$ & & & \\
\hline
\end{tabular}

These results indicate that using 18-flute tungsten carbide bur produces less enamel surface roughness in comparison to debonding pliers. Regarding the comparison between group one (18-flute tungsten carbide bur) and group two (12flute tungsten carbide bur) and also between group two (12-flute tungsten carbide bur) and group three (adhesive removal plier), there was no statistically significant difference.

\section{Discussion}

Clinical orthodontic treatment has been revolutionized during the past decades with the advent of direct bonding. Placement of orthodontic attachments on the surface of teeth can be accomplished by using bonding materials. However, unlike when used for restorative dentistry, these materials must be removed from the surface of enamel after orthodontic treatment. The concern over debonding-induced enamel surface alterations derives from the importance of the uppermost layer of enamel attributed to its hardness, higher mineral content and more fluoride relative to deeper zones. ${ }^{7}$

The removal of orthodontic brackets aims to separate not only the base of the bracket from the tooth as well as any remaining resin to restore the same condition before orthodontic treatment. However, this is not always possible and may lead to mechanical removal of the enamel, endangering healthy dental structure and, providing irreversible damage to the enamel. This damage can be reduced depending on the technique used for bracket removal. Among these pliers for the mechanical removal, different diamond burs of high and low speed, abrasive discs, rubber tips, ultrasonic units, and air abrasion techniques are among the most commonly used techniques. 9,10

Debonding and cleanup are operatordependent procedures, therefore the results might vary between operators. To minimize this error, only one operator carried out all the clinical procedures in the present study. Results of the present study revealed that post bonding enamel surface roughness was more than the pre bonding enamel surface roughness in all the groups. This means that no clean-up method was able to fully restore the enamel surface roughness to its original state. ${ }^{1}$ This study evaluated enamel surfaces after debonding with the use of traditional resin removal procedures. Clean-up method efficiency was also checked in our study and, we found that debonding with adhesive removing plier was least efficient followed 12-fluted flame -shaped carbide bur and 18-fluted flameshaped tungsten carbide bur, so the best clean-up method in this study achieved is by using 18-fluted flame-shaped tungsten carbide bur. This finding was evaluated by keeping the other factors constant for example type of adhesive, types of brackets, type of debonding plier, etc.

Graber et al. ${ }^{11}$ stated that the most important objective of adhesive removal is leaving a smooth enamel surface. The18fluted flame-shaped tungsten carbide bur 
eliminated all of the problems experienced with the 12-fluted flame-shaped carbide burs and the adhesive removal plier at the parameters tested. The flat sides of the bur allowed for more surface area contact with the enamel, and the increased number of flutes with the decreased distance between blades (less than $150 \mu \mathrm{m}$ ). Both of these factors allowed for the distribution of the rotary forces over a greater area and more blades resulting in a smoother overall enamel surface than the other tested methods. Palmer et al., ${ }^{6}, 2018$ stated that the 20-fluted flame-shaped tungsten carbide bur on high speed resulted in the smoothest enamel surface qualitatively and coinciding with our findings. Our finding was similar to the findings of Goel et al. ${ }^{1}$ (2017), who reported that Sof-Lex discs produce less enamel loss than the 12-fluted flame-shaped high-speed tungsten carbide bur. Also, our finding was similar to the findings of Krell et al. ${ }^{12}$ who reported that the high speed 12-fluted flame-shaped tungsten carbide bur produces more enamel surface loss than the ultrasonic scaler.

Zarrinia et al. ${ }^{13}$ stated that carbide burs at high speed proved to be efficient in residual resin removal but failed to produce a satisfactory enamel surface. This finding is similar to our findings. Meira Cardoso et al., ${ }^{14} 2014$ reported that using 30-blades tungsten carbide bur at high speed after bracket debonding with polishing reduced the enamel surface roughness when compared to initial roughness and this finding is in contrast with our findings. Nevertheless, the TCB method had a minimal level of roughness, ${ }^{15}$ and proved to be an effective method ${ }^{16}$ that causes less damage with faster performance. ${ }^{17}$ Conversely, Karan et al., ${ }^{18}$ as well as Tavares ${ }^{19}$, asserted that this method, when compared to fiberglass burs as well as other methods, presents increased roughness.Meira Cardoso et al, ${ }^{14}$, Pignatta et $a l^{20}$ and Miksic et al, ${ }^{21}$, who described that using of adhesive removing plier for adhesive remnant removal on enamel surface as the worst choice because those visible injuries were caused due to enamel surface convexity while the plier was being supported by the occlusal surface to allow the flat active tip to remove residual adhesive by compression and these findings were similar with our findings.

In contrast, Hosein et $a l^{22}$ Albuquerque et al. ${ }^{16}$ and Tavares ${ }^{19}$ who, stated that using adhesive removal plier produced little enamel roughness and demanded minimal time for reducing adhesive remnant, and these findings were dissimilar with our findings.

Both Eliades et al., ${ }^{7}$ and Ahrari et al., ${ }^{15}$ were used tungsten carbide burs in low and high speed and reported that this method of removing of adhesive remnant removal was better and, the best choice than the other methods and not caused irreversible damage to the enamel surface, and these findings were similar with our findings.

Thus, orthodontists should attempt to choose a suitable protocol based on scientific evidence for adhesive remnant removal and, initial tooth features restoration to avoid undesirable results, reach professional and patient's goals and ensure satisfactory conservative, successful treatment outcomes. ${ }^{23,24}$

\section{Conclusion}

It can be concluded from the study that: No clean-up procedure was able to restore the enamel to its original smoothness. All adhesive remnant removal methods changed enamel surface roughness. Rotary instruments that have the ability to remove residual resin cement bonded to the enamel surface without providing excessive damage to the tooth structure should be preferred. The 18-fluted flame-shaped tungsten carbide bur at high speed for orthodontic adhesive removal demonstrated more favorable results in our hands, as it resulted in the smoothest enamel surface and could reasonably be used as a standard by which future other burs or other procedures are compared. The use of the adhesive removal plier as employed in this study is not well suited for adhesive removal in orthodontic debonding procedures as tested due to resulting increased roughness of the tooth enamel and decreased efficiency of the removal procedure.

\section{Conflict of interest}

The authors reported no conflict of interests. 


\section{References}

1-Goel A, Singh A, Gupta T, Gambhir RS. Evaluation of surface roughness of enamel after various bonding and clean-up procedures on enamel bonded with three different bonding agents: $A n$ invitro study. J Clin Exp Dent.2017;9(5):e608-16.

2-Uctasli MB, Arisu HD, Omurlu H, Eliguzeloolu E, Ozcan S, Ergun $\mathrm{G}$. The effect of different finishing and polishing systems on the surface roughness of different composite restoration materials. J Contemp Dent Pract.2007; 8:89-96.

3-Ozer T, Basaran A, Kama JD. Surface roughness of the restored enamel after orthodontic treatment. Am J Orthod Dentofacial Orthop.2010;137:368-74.

4-VidorMM, Felix RP, Marchioro EM, Hahn L.Enamel surface evaluation after bracket debonding and, different resin removal methods. Dental Press J Orthod.2015 Mar-Apr;20(2):61-7.

5- Mahdi HA, Ghaib NH, Saloom HF. Evaluation of enamel surface damage after debonding using three different pliers "An in vitro study". MDJ 2011;8(3):281-287

6. Palmer JA, Mang T, Tabbaa S, Al-Jewair T. Analysis of enamel surface roughness after different adhesive removal techniques for orthodontic bracket debonding. Lasers in Dental Science. 2018; 2:95101.

7. Eliades T, Gioka C, Eliades G, Makou M. Enamel surface roughness following debonding using two resin grinding methods. Eur J Orthod. 2004; 26:333-8.

8. Ahmed T, Rahman NA, Alam M. Assessment of in vivo bond strength studies of the orthodontic bracket-adhesive system: A systematic review. Eur J Dent. 2018; 12(04): 602-9.

9. Campbell PM. Enamel surfaces after orthodontic bracket debonding. Angle Orthod 1995; 65:10310.

10. Bandeca M, Porto TS, Frizzera F, de Andrade MF. Methods for removal of resin remaining after debonding of orthodontic brackets: A literature review. Journal of Dental Research and Review 2014; 1(2):105-107.

11. Graber LW, Vanarsdall RL, Vig KWL (2012) Orthodontics current principles and techniques, 5th ed. Mosby, Philidelphia.

12. Krell KV, Courey JM, Bishara SE. Orthodontic bracket removal using conventional and ultrasonic debonding techniques, enamel loss, and time requirements. Am J Orthod Dentofacial Orthop. 1993; 103:258-66.

13. Zarrinia K, Eid NM, Kehoe MJ. The effect of different debonding techniques on the enamel surface: An in vitro qualitative study. Am J Orthod Dentofacial Orthop. 1995;108:284-93.

14. Meira Cardoso LAM, Valdrighi HC, Filho MV, Correr $A B$. Effect of adhesive remnant removal on enamel topography after bracket debonding. Dental Press J Orthod. 2014 Nov-Dec;19(6):105-12.

15-Ahrari F, Akbari MJ, Dabiri G.Enamel surface roughness after debonding of orthodontic brackets and various clean-up techniques.J Dent (Tehran) 2013;10:82-93.

16. Albuquerque GS, Vedovello Filho $M$, Lucato AS, Boeck EM, Degan V, Kuramae M. Evaluation of enamel roughness after ceramic bracket debonding and clean-up with different methods. Braz J Oral Sci. 2010; 9(2):81-4.

17. Mahdavie NN. The Effect of various debonding burs on the enamel surfaces of teeth after debonding metal brackets [tese]. Chicago: University of Illinois; 2012.

18. Karan S, Kircelli BH, Tasdelen B. Enamel surface roughness after debonding: comparison of two different burs. Angle Orthod. 2010; 80(6):1081-8.

19. Tavares SW. In vitro analysis of different methods of removal of residual resin in dental enamel [thesis]. Piracicaba (SP): State University of Campinas; 2006.

20. Pignatta LMB, Duarte Jr S, Santos ECA. Evaluation of enamel surface after bracket debonding and polishing. Dental Press J Orthod. 2012; 17(4):7784

21. Miksic M, Slaj M, Mestrovic S. Qualitative analysis of the enamel surface after removal of remnant composite. Acta Stomatol Croat. 2003;37 (3):247-51.

22. Hosein I, Sheirriff M, Ireland AJ. Enamel loss during bonding, debonding, and clean up with the use of a self-etching primer. Am J Orthod Dentofacial Orthop. 2004; 126(6):717-24.

23. Macieski K, Rocha R, Locks A, Ribeiro GU. Effects evaluation of remaining resin removal (three modes) on enamel surface after bracket debonding. Dental Press J Orthod. 2011;16(5):146-54.

24. Zanarini M, Gracco A, Lattuca M, Marchionni S, Gatto MR, Bonetti A. Bracket base remnants after orthodontic debonding. Ang/e Orthod. 2013; 83 (5):885-91. 